\title{
Intramyocellular lipid and its relationship with insulin sensitivity and fat in type 2 diabetic men of White and Black ethnicity
}

\author{
O. Hakim ${ }^{1,2}$, G. Charles-Edwards ${ }^{2}$, B. Whitcher ${ }^{3}$, H. Shuaib ${ }^{3}$ and L.M. Goff ${ }^{1}$ \\ ${ }^{1}$ Division of Diabetes and Nutritional Sciences, \\ ${ }^{2}$ Department of Biomedical Engineering, King's College London, SE1 IUL, UK and \\ ${ }^{3}$ Klarismo Ltd
}

This abstract was presented as the Whole Body Metabolism Theme Highlight

Lipid accumulation in skeletal muscle cells, termed intramyocellular lipids (IMCL), is inversely associated with insulin sensitivity, independent of overall adiposity, and is elevated in populations prone to developing type 2 diabetes (T2D) and in those with T2 $\mathrm{D}^{(1)}$. Populations of African descent are disproportionately affected by T2D but there has been limited investigation of IMCL $^{(2)}$. This study aimed to compare IMCL and extramyocellular lipid (EMCL) between men of White European (WE) and Black West African (BWA) ethnicity with T2D, and to investigate relationships between IMCL, insulin sensitivity (IS) and adiposity measures.

In a cross-sectional study of $19 \mathrm{WE}$ and 18 BWA men with T2D, soleus IMCL content was determined using proton-magnetic resonance spectroscopy $\left({ }^{1} \mathrm{H}-\mathrm{MRS}\right)$, skeletal muscle IS using a hyperinsulinaemic-euglycaemic clamp with infusion of [6,6 ${ }^{2} \mathrm{H}_{2}$ ]-glucose for quantification of peripheral glucose disposal, and whole-body fat (\%) using magnetic resonance imaging (MRI). ${ }^{1}$ H-MRS data was analysed using Java-based Magnetic Resonance User Interface (jMRUI) software. MRI data was analysed by an automated quantitative method (Klarismo Ltd).

The ethnic groups were well matched for age, weight and BMI (all $p>0 \cdot 05$ ). There were no ethnic differences in IMCL, EMCL, IS or $\%$ whole-body fat, Table 1 . Correlation analysis showed IMCL was not significantly associated with IS in either ethnic group (WE: $\mathrm{r}=-0.118, p=0.642$; BWA: $\mathrm{r}=-0 \cdot 301, p=0 \cdot 225)$. Correlations of IMCL with adiposity measures showed IMCL was associated with $\%$ whole-body fat in BWA men $(r=0.477, p=0.039)$ but not WE men $(r=0.163, p=0.533)$. Also, IMCL was associated with EMCL in BWA men $(\mathrm{r}=0.754, p<0.001)$ but not WE men $(\mathrm{r}=0.079, p=0.754)$ and EMCL was associated with waist circumference in WE men $(\mathrm{r}=0.486, p=0.041)$ but not BWA men $(\mathrm{r}=0 \cdot 229, p=0 \cdot 346)$.

Table 1. Characteristics of White European and Black West African men

\begin{tabular}{|c|c|c|c|c|c|}
\hline & \multicolumn{2}{|c|}{$\mathrm{WE}(\mathrm{n}=18)$} & \multicolumn{2}{|c|}{ BWA $(n=19)$} & \multirow[t]{2}{*}{$P$-value } \\
\hline & Mean & SD & Mean & SD & \\
\hline Age (years)* & 58 & 6 & 54 & 11 & $0 \cdot 473$ \\
\hline Weight (kg) & $99 \cdot 8$ & $16 \cdot 7$ & $92 \cdot 7$ & 12 & $0 \cdot 148$ \\
\hline BMI $\left(\mathrm{kg} / \mathrm{m}^{2}\right)$ & $31 \cdot 5$ & $4 \cdot 1$ & $30 \cdot 1$ & $3 \cdot 6$ & $0 \cdot 259$ \\
\hline$\%$ Whole-body fat $\dagger$ & $30 \cdot 1$ & 5.9 & $26 \cdot 6$ & $5 \cdot 7$ & 0.082 \\
\hline Whole-body visceral fat $(\mathrm{ml})^{\wedge \dagger}$ & 6309 & $5129-7762$ & 4169 & $3548-4898$ & $0 \cdot 003$ \\
\hline Insulin sensitivity $\left(\mu \mathrm{mol} / \mathrm{m}^{2}\right.$ BSA $\left.\min ^{-1}\right) \ddagger$ & 955 & 379 & 1132 & 418 & $0 \cdot 192$ \\
\hline $\operatorname{IMCL}(A U)^{\wedge}$ & $0 \cdot 039$ & $0.032-0.048$ & $0 \cdot 044$ & $0 \cdot 033-0 \cdot 058$ & $0 \cdot 507$ \\
\hline EMCL (AU)* & $0 \cdot 047$ & 0.028 & $0 \cdot 049$ & $0 \cdot 033$ & 0.855 \\
\hline
\end{tabular}

Data presented as geometric mean $(95 \% \mathrm{CI})$ for $\log$ transformed data $\left(^{*}\right)$ or median (interquartile range) for non-parametric data $\left({ }^{\wedge}\right) . P$ values determined using

independent samples t-tests for normally distributed data otherwise Mann-Whitney test. $\uparrow$ WE: $n=17, B W A: n=19 ;+$ WE: $n=18$, BWA: $n=18$

There were no ethnic differences in IMCL, and it was not associated with IS in either ethnic group however the association between IMCL and measures of adiposity differed between the ethnic groups. IMCL was associated with whole-body adiposity and EMCL in BWA but not WE men suggesting that IMCL accumulation may be independent of whole-body adiposity in WE men with T2D and could be influenced by other factors.

1. Sinha R, Dufour S, Petersen KF et al. (2002) Diabetes 51, 1022-7.

2. Ingram KH, Lara-Castro C, Gower BA et al. (2011) Obesity (Silver Spring) 19, 1469-75. 